\title{
Neorazzismo, neoschiavismo
}

Il razzismo in Europa (e in Italia) è una realtà: lo attestano casi concreti e documentati che ormai non rappresentano più l'eccezione o qualcosa di straordinario, nonché alcuni rapporti internazionali come quello dell'ILO, l'Agenzia per il Lavoro dell'Onu (6 marzo 2009), o quello del Commissario per i diritti umani del Consiglio d'Europa (15 gennaio 2009).

Ciò significa fare i conti, ancora e di nuovo, con la costruzione, lo sviluppo, gli esiti perversi delle identità, collettive e sociali.

Come ha osservato recentemente Gianfrancesco Zanetti, «ogni identità ha la sua storia. Talvolta, il senso della propria identità collettiva è addirittura basato su elementi percepiti come fisici, biologici, di sangue: in tal senso il primo manifesto, compiuto ed esplicito, esito comunque di una stratificazione di processi, del razzismo cosiddetto scientifico può essere considerato il Saggio sull'ineguaglianza delle razze umane di Joseph Arthur de Gobineau. D'altro canto, si potrebbe sostenere, per fare un esempio in negativo, che l'identità italiana non ha avuto, originariamente, una base razzista: il "noi" italiano non si è costituito sullo sfondo di un contrasto con un'alterità concepita in termini biologici. Da questo punto di vista, la storia dell'identità italiana è radicalmente diversa da quella, per esempio, americana». E tuttavia anche l'identità italiana - aspetto spesso negletto e oscurato - è attraversata da ricorrenti insorgenze di natura razziale e razzista.

Imprevedibili e improvvisi fenomeni che si sono determinati a partire dai primi anni novanta del Novecento - flussi migratori, globalizzazione economica (e sua recente, strutturale, crisi), irruzione di nuovi media come la rete informatica, irrigidimenti ideologici e nuovi fondamentalismi-hanno segnato, come ha spiegato Etienne Balibar, il «ritorno al futuro della razza». La necessità di un'omogeneità che sta al fondo degli assetti istituzionali, di un idem sentire, di shared values, ha generato il recupero di uno «schema genealogico»-Balibar lo mostra nel suo contributo a questo fascicolo - che si interseca con il modo di intendere la cultura stessa, il diritto, la politica istituzionale. Rispetto a fenomeni che - entro una interpretazione divenuta egemone - avrebbero portato il caos in un (supposto) ordine, costituitosi nel tempo, e avrebbero messo in questione certezze impor- 
tanti (e ciò nasconde, in realtà, il disordine generato da profondi squilibri economici e sociali, dalle enormi carenze della politica nel dare risposte ai vecchi e nuovi bisogni delle persone), rispetto al disagio e allo smarrimento, si determinano risposte di matrice neorazzista, incoraggiate e in parte addirittura costruite da alcune forze politiche in vari paesi dell'Europa. Si genera così un 'circolo vizioso' preoccupante fra il discorso e l'azione dei governi mentre il sistema mediatico contribuisce all'opera di riproduzione e diffusione di stereotipi e pregiudizi.

Le inquietudini, le aspirazioni, le rivendicazioni, la denuncia dei bisogni, lungi dall'indirizzarsi verso forme di partecipazione, mobilitazione, protesta, conflitto nello spazio pubblico-politico, sono incanalate verso un bersaglio: gli 'estranei', $i$ 'diversi', gli 'stranieri', gli 'extracomunitari', gli 'irregolari', $i$ 'clandestini', $i$ 'negri'. La logica del «capro espiatorio», legittimata dagli imprenditori mediatici e politici del razzismo, assale le istituzioni conducendo a provvedimenti e scelte sia a livello micro sia a livello macro che fanno regredire la politica democratica e inclusiva: dalle ordinanze comunali allo stato di emergenza, si attuano scelte che configurano un diritto speciale riservato ai migranti e alle minoranze. La logica securitaria e discriminatoria domina la scena, riorganizzando nel profondo, oltre che il senso comune, l'identità collettiva. La logica dell'esclusione e della gerarchizzazione connessa all'idea della razza - di cui Gobineau, e con altre modalità, Evola costituiscono gli archetipi - ritorna, strutturando la dimensione sociale del razzismo odierno. Si tratta di un sistema, spesso subdolo, di disuguaglianze giuridiche, economiche e sociali, caratterizzato da forti scarti di potere fra i gruppi coinvolti. $\grave{E}$ un sistema - come ha puntualmente osservato Annamaria Rivera - che si costituisce e si rafforza mediante una pluralità di strategie: la strategia dell'esclusione, simbolica e sociale, che recupera appunto anche le tesi razziste della modernità (lo suggerisce Mara Fornari nel suo contributo); la strategia dell'espulsione o della segregazione permanente di cui i Centri per richiedenti asilo (CARA), in Italia, rappresentano una forma concreta (come illustra Chiara Marchetti indagando la nuova politica dei campi diffusasi in Occidente negli ultimi anni); la strategia dell'inclusione differenziata a scopi strumentali, e qui si pensi alla collocazione di certe 'categorie' di persone ridotte a semplici 'braccia da lavoro' o addirittura a nuovi schiavi, e dunque duramente e violentemente discriminate: si è dinanzi a un modulo che ha avuto in Australia, fra Ottocento e Novecento, una peculiare configurazione con il fenomeno del blackbirding (qui indagato da Gaia Giuliani), in cui si combinano assegnazione razzista del colore e lavoro servile, e che oggi trova fondamento nella "gerarchia globale delle cittadinanze» e in una specifica metamorfosi dell'idea stessa di «integrazione» (come spiega Dino Costan- 
tini). Neorazzismo e neoschiavismo si coalizzano così nel violare, in modo palese o subdolo, l'intima essenza di individui e gruppi.

Il razzismo grossolano, quello più evidente, ha alimentato nel tempo anche un razzismo 'rispettabile', 'democratico', che permea l'elettorato ma anche alcuni settori dei gruppi dirigenti dei partiti che dovrebbero, richiamandosi alle carte costituzionali, essere gli strenui difensori dei diritti fondamentali per ogni persona. Sta qui, in fondo, la gravità della 'questione razziale', oggi, in Europa e in Italia: l'intreccio tra razzismo di Stato (o istituzionale), razzismo mediatico e razzismo popolare. Il lessico e le pratiche razziste ridisegnano così, insieme all'identità dei popoli, anche le politiche pubbliche e le condizioni del lavoro. Ma è alla politica stessa, intesa come impegno collettivo capace di trasformare $i$ conflitti in "progetti di liberazione» (riprendendo ancora Annamaria Rivera), che va affidato «il tentativo di andare oltre l'ordine presente» e di costruire una società ove il 'noi' si costruisca non per opposizione ma in relazione agli 'altri'. Comprendere a fondo certi fenomeni e alcune linee di tendenza che li originano e li giustificano, può costituire, sotto questo profilo, l'inizio di un lungo lavoro.

(th.c.)

Tutte le immagini riprodotte in questo numero sono tratte dal servizio fotografico realizzato da Federico M. Picciani nel novembre 2010 in Malawi. 